\title{
Impact of Temperatures on Hessian Fly (Diptera: Cecidomyiidae) Resistance in Selected Wheat Cultivars (Poales: Poaceae) in the Great Plains Region
}

\author{
MING-SHUN CHEN,${ }^{1,2,3}$ SHANDA WHEELER, ${ }^{2}$ HAIYAN WANG,${ }^{4}$ AND R. JEFFREY WHITWORTH ${ }^{2}$
}

\section{J. Econ. Entomol. 107(3): 1266-1273 (2014); DOI: http://dx.doi.org/10.1603/ECl3357}

\begin{abstract}
Changes in temperature can result in fundamental changes in plant physiology. This study investigated the impact of different temperatures from 14 to $26^{\circ} \mathrm{C}$ on the resistance or susceptibility to the Hessian fly, Mayetiola destructor (Say), of selected wheat cultivars that are either currently popular in the Great Plains area or soon to be released to this region. We found that many wheat cultivars including 'Bill Brown,' 'Byrd,' 'Endurance,' 'Fuller,' 'GA-031257-10LE34,' and 'KS09H19-2-3' were susceptible to Hessian fly infestation at $\geq 20^{\circ} \mathrm{C}$, but became resistant at a certain lower temperature, depending on different cultivars. These cultivars were classified as Hessian fly susceptible according to the traditional standards, and their impact on Hessian fly management needs to be reevaluated. However, many wheat cultivars that were resistant at $\leq 20^{\circ} \mathrm{C}$ became destabilized at a certain higher temperature. Phenotypic variations among the resistant cultivars at different temperatures were also observed, suggesting potential different resistance mechanisms. Studies on the genetic and molecular mechanisms associated with resistance at different temperatures are needed, which may lead to improved wheat cultivars with more durable resistance to Hessian fly infestation.
\end{abstract}

KEY WORDS Hessian fly, Mayetiola destructor, plant resistance, temperature effect, wheat

The Hessian fly, Mayetiola destructor (Say), is a serious pest of wheat in the United States and worldwide (Hatchett et al. 1987, Buntin 1999, Pauly 2002, Stuart et al. 2012). Since its introduction to the United States during the American Revolution $\approx 1,779$, major Hessian fly outbreaks have been recorded, and localized serious damage due to this pest occurs every year. In recent years, heavily infested fields have occurred more frequently and on a larger scale, especially in the southern part of the United States, including Oklahoma, Texas, Georgia, and Louisiana (Colyer et al. 1989, Royer 2005, Watson 2005, Comis 2007, Knutson and Swart 2007, Smith 2007, Huang et al. 2011). This recent increase in Hessian fly incidence may be a result of climate change or the widely adopted no-till cultivation practice.

Major control measures for the Hessian fly include 1) late planting (Best Pest Management Planting Date) to avoid infestation from the fall generation; 2) timely destruction of volunteer wheat; 3 ) seed treatment with systemic pesticides; and 4) deployment of resistant wheat cultivars (Buntin and Bruckner 1990,

\footnotetext{
${ }^{1}$ Hard Winter Wheat Genetics Research Unit, Center for Grain and Animal Health, USDA-ARS, 1515 College Ave., Manhattan, KS 66506.

2 Department of Entomology, Kansas State University, 123 Waters Hall, Manhattan, KS 66506.

${ }^{3}$ Corresponding author, e-mail: mchen@ksu.edu or mingshun.chen@ars.usda.gov.

${ }^{4}$ Department of Statistics, Kansas State University, 101 Dickens Hall, Manhattan, KS 66506.
}

Zelarayan et al. 1991, Buntin 1992, Buntin et al. 1992). Each control measure has its limitations. Late planting can only be adopted in cooler wheat growing regions because Hessian fly emergence can occur in late fall in warmer regions. In addition, many farmers plant wheat early for cattle grazing. Destruction of volunteer wheat is hard to coordinate within large wheat growing areas. The effect of seed treatment lasts only for 2-4 wk. Chemical application beyond seed treatment is generally not effective because of the nature of Hessian fly damage. Hessian fly larvae are hard to see because they are very small and live inside wheat plants. Once the damage is visible, it is too late to apply chemical pesticides because the damage is irreversible (Byers and Gallun 1971). Deployment of resistant cultivars is highly effective and cost efficient. However, resistance conferred by specific resistance genes is usually short-lived, lasting for only $6-8 \mathrm{yr}$ (Gould 1998).

To date, 34 Hessian fly resistance genes have been identified and are named as $H 1-H 34$ (Li et al. 2013). All known resistance genes are inherited as major dominant traits except $h 4$, which is recessive, $H 7 H 8$, which are dominant but must be inherited together to be effective (Amri et al. 1990), and H34, which is a newly identified gene that has not yet been well characterized ( $\mathrm{Li}$ et al. 2013). All resistance genes are antibiotic to Hessian fly larvae, so that larvae die within plants without developing into second-instar larvae (Stuart et al. 2012). Wheat genes with resis- 
tance to Hessian fly are temperature-sensitive. Resistance is lost when temperature is above a certain degree depending on different resistance genes (Sosa and Foster 1976, Sosa 1979, Tyler and Hatchett 1983, Ratanatham and Gallun 1986). Among the known resistance genes, H18 is the most sensitive, and it loses resistance when air temperature is $>18^{\circ} \mathrm{C}$ (Cambron et al. 1996). Other known resistance genes are also temperature-sensitive, but the resistance remains as long as temperature is $<22^{\circ} \mathrm{C}$ (Buntin et al. 1990). While screening wheat breeding lines for Hessian fly resistance, we observed that some lines were highly sensitive to changes in greenhouse temperatures, and often yield very inconsistent resistance results to Hessian fly from year to year. The objectives of this study were to determine the ranges of temperatures under which selected elite wheat cultivars in the Great Plains lose resistance; and to determine the duration under these temperatures to lose or gain resistance. We observed that many wheat cultivars that were categorized as susceptible previously are actually resistant under lower temperatures.

\section{Materials and Methods}

Hessian Fly. The Great Plains (GP) Hessian fly biotype was used in this study. Biotype GP was derived from a colony collected from Ellis County, KS, in 1988 (Gagne and Hatchett 1989). Since then, the insects have been maintained on susceptible wheat seedlings ('Newton' or 'Karl 92') in the greenhouse at $20^{\circ} \mathrm{C}$. Biotype GP is avirulent to all known major Hessian fly resistance genes (Ratcliffe et al. 1994).

Wheat Cultivars. Each year scientists at the Kansas State Experimental Station, Kansas State University, Manhattan, KS, assemble a set of recently released or soon-to-be-released wheat cultivars from public and private breeders in the Great Plains area for testing resistance to various diseases and insects. Fifty-seven wheat cultivars from the 2013 set were used to study the impact of different temperatures on wheat resistance to Hessian fly. In addition to the 57 wheat cultivars, cultivars Newton and Karl92 were also used as susceptible controls in all experiments.

Infestation and Phenotyping. Approximately 12 wheat seeds were planted in each test. Wheat lines were planted in flats ( 54 by 36 by $8 \mathrm{~cm}$ ) filled with PRO-MIX 'BX' potting mix (Hummert Inc., Earth City, MO). Each flat was divided into 24 sections with a divider and each cultivar was planted into each section. The flats were initially placed in a greenhouse to geminate. After $7 \mathrm{~d}$, when the second leaf was beginning to emerge, the plants were infested with Hessian fly eggs by confining fly adults in a tent that enclosed the wheat seedlings. Female flies oviposit on the adaxial surface of plants in a free-choice manner, and eggs hatch in 3-5 d, depending on temperatures. When egg density reached $\approx 8$ per plant, adult flies were removed from the tent, and the flats were transported into growth chambers programmed at various temperatures with a photoperiod of 14:10 (L:D) h. Neonate larvae migrate along the leaf, enter into a plant, and live between the first and second leaf under this condition.

Twenty-one days after infestation, plants were categorized and recorded as susceptible or resistant. During phenotyping, the first leaf sheath was separated from the second leaf sheath, and the dissected plant was examined under a microscope. Dead larvae (reddish and skinny) can be easily distinguished from live larvae (white and fat) by differences in color and size. Plants were considered resistant if they contained dead larvae and had grown normally. Plants were considered susceptible if they contained live larvae and were stunted. Plants with no dead or live larvae were categorized as escapes.

Temperature Duration Test. For cultivars requiring temperatures $<20^{\circ} \mathrm{C}$ to exhibit Hessian fly resistant trait, we determined the duration of lower temperatures necessary to exhibit resistance. Wheat seedlings were infested as stated previously, seedlings with eggs were initially cultured at $20^{\circ} \mathrm{C}$ for $3 \mathrm{~d}$, and the temperature was adjusted to $14^{\circ} \mathrm{C}$ before egg hatching. Egg hatch and larval migration were monitored on an hourly basis to determine the time when larvae reached the feeding site. When an average of four larvae reached the feeding site on a plant, the time was recorded as the initial attack time (Time 0 ). Seedlings were continuously cultivated under $14^{\circ} \mathrm{C}$ for $12,24,48$, 72 , and $120 \mathrm{~h}$, and then transferred to a growth chamber at $20^{\circ} \mathrm{C}$ until scoring.

Statistical Analyses. Data were fitted to a logistic regression model using the numbers of resistant and susceptible plants as the response variable and temperature and wheat cultivar as the explanatory variables (factors). A deviance test with chi-square distribution was used to obtain $P$ values. For each cultivar tested, a logistic model with deviance test was also used to assess whether the percentage of resistant plants differed significantly across different temperatures. After Bonferroni (1935) correction, a test was considered significant at the 0.05 level if the $P<0.0028$ for cultivars tested at lower temperatures; and if the $P<0.0011$ for cultivars tested at higher temperatures.

\section{Results}

Phenotypes of Selected Wheat Cultivars at $20^{\circ} \mathrm{C}$. Cultivars were first tested for Hessian fly resistance at $20^{\circ} \mathrm{C}$, the temperature at which routine screening was carried out. Of the 57 cultivars, 41 had $\geq 90 \%$ resistance (Table 1). 'Everest' showed 77\% resistance, cultivars including 'Hatcher,' 'GA-031257-10LE34,' 'WB-Redhawk,' 'KS09H19-2-3,' 'Bill Brown,' and 'Thunder CL' had 10-40\% resistance, and cultivars including 'Danby,' 'Brawl CL Plus,' 'Byrd,' 'Denali,' 'Endurance,' 'Fuller', 'GA-045710-10E46, 'LCSMint,' and 'Santa Fe' had $<10 \%$ resistance. The susceptible control cultivars Newton and Karl92 had 0\% resistance.

Some Cultivars Susceptible at $\geq 20^{\circ} \mathrm{C}$ Become Resistant at Lower Temperatures. Cultivars with $<90 \%$ resistant plants at $20^{\circ} \mathrm{C}$ were retested at $14^{\circ} \mathrm{C}$ (range of $\left.14-16^{\circ} \mathrm{C}\right), 16^{\circ} \mathrm{C}\left(16-18^{\circ} \mathrm{C}\right)$, and $18^{\circ} \mathrm{C}\left(18-20^{\circ} \mathrm{C}\right)$. As shown in Table 2 , the impact of lower temperatures 
Table 1. Resistance or susceptibility of selected wheat cultivars to Hessian fly from the United States Great Plains at $20-22^{\circ} \mathrm{C}$

\begin{tabular}{|c|c|c|c|c|c|c|c|c|c|c|c|c|c|}
\hline \multirow{2}{*}{ Cultivar } & \multicolumn{3}{|c|}{ Replicate 1} & \multicolumn{3}{|c|}{ Replicate 2} & \multicolumn{3}{|c|}{ Replicate 3} & \multicolumn{3}{|c|}{ Total } & \multirow{2}{*}{$\mathrm{SD}$} \\
\hline & $\mathrm{R}$ & $\mathrm{S}$ & $\overline{\% \mathrm{R}}$ & $\mathrm{R}$ & $\mathrm{S}$ & $\overline{\% R}$ & $\mathrm{R}$ & $\mathrm{S}$ & $\% \mathrm{R}$ & $\mathrm{R}$ & $\mathrm{S}$ & $\% \mathrm{R}$ & \\
\hline 1863 & 11 & 0 & 100 & 8 & 0 & 100 & 10 & 0 & 100 & 29 & 0 & 100 & 0 \\
\hline 2135 & 11 & 0 & 100 & 11 & 0 & 100 & 12 & 0 & 100 & 34 & 0 & 100 & 0 \\
\hline 2166 & 10 & 0 & 100 & 9 & 0 & 100 & 10 & 0 & 100 & 29 & 0 & 100 & 0 \\
\hline 2525 & 11 & 0 & 100 & 10 & 0 & 100 & 8 & 0 & 100 & 29 & 0 & 100 & 0 \\
\hline $25 \mathrm{R} 30$ & 10 & 0 & 100 & 9 & 0 & 100 & 8 & 0 & 100 & 27 & 0 & 100 & 0 \\
\hline $25 \mathrm{R} 32$ & 11 & 0 & 100 & 7 & 0 & 100 & 11 & 0 & 100 & 29 & 0 & 100 & 0 \\
\hline 25R39 & 10 & 0 & 100 & 10 & 0 & 100 & 9 & 0 & 100 & 29 & 0 & 100 & 0 \\
\hline Armour & 7 & 0 & 100 & 11 & 0 & 100 & 8 & 0 & 100 & 26 & 0 & 100 & 0 \\
\hline Art & 12 & 0 & 100 & 11 & 0 & 100 & 11 & 0 & 100 & 34 & 0 & 100 & 0 \\
\hline Billings & 11 & 0 & 100 & 11 & 0 & 100 & 12 & 0 & 100 & 34 & 0 & 100 & 0 \\
\hline Centerfield & 10 & 0 & 100 & 8 & 0 & 100 & 9 & 0 & 100 & 27 & 0 & 100 & 0 \\
\hline CJ & 11 & 0 & 100 & 9 & 0 & 100 & 12 & 0 & 100 & 32 & 0 & 100 & 0 \\
\hline Clara CL (W) & 9 & 0 & 100 & 9 & 0 & 100 & 11 & 0 & 100 & 29 & 0 & 100 & 0 \\
\hline Deliver & 7 & 0 & 100 & 9 & 0 & 100 & 11 & 0 & 100 & 27 & 0 & 100 & 0 \\
\hline Duster & 10 & 0 & 100 & 11 & 0 & 100 & 8 & 0 & 100 & 29 & 0 & 100 & 0 \\
\hline GA-031086-10E26 & 12 & 0 & 100 & 10 & 0 & 100 & 11 & 0 & 100 & 33 & 0 & 100 & 0 \\
\hline Gallagher & 12 & 0 & 100 & 9 & 0 & 100 & 11 & 0 & 100 & 32 & 0 & 100 & 0 \\
\hline Garrison & 7 & 0 & 100 & 11 & 0 & 100 & 8 & 0 & 100 & 26 & 0 & 100 & 0 \\
\hline Greer & 10 & 0 & 100 & 12 & 0 & 100 & 8 & 0 & 100 & 30 & 0 & 100 & 0 \\
\hline Jackpot & 9 & 0 & 100 & 11 & 0 & 100 & 10 & 0 & 100 & 30 & 0 & 100 & 0 \\
\hline LCH08-109 & 11 & 0 & 100 & 8 & 0 & 100 & 10 & 0 & 100 & 29 & 0 & 100 & 0 \\
\hline LCH08-80 & 12 & 0 & 100 & 9 & 0 & 100 & 11 & 0 & 100 & 32 & 0 & 100 & 0 \\
\hline LCH09-19 & 10 & 0 & 100 & 11 & 0 & 100 & 9 & 0 & 100 & 30 & 0 & 100 & 0 \\
\hline LCS08-12 & 10 & 0 & 100 & 9 & 0 & 100 & 11 & 0 & 100 & 30 & 0 & 100 & 0 \\
\hline OK09915C & 11 & 0 & 100 & 9 & 0 & 100 & 10 & 0 & 100 & 30 & 0 & 100 & 0 \\
\hline PostRock & 8 & 0 & 100 & 11 & 0 & 100 & 10 & 0 & 100 & 29 & 0 & 100 & 0 \\
\hline SY Southwind & 9 & 0 & 100 & 11 & 0 & 100 & 8 & 0 & 100 & 28 & 0 & 100 & 0 \\
\hline $\mathrm{T} 153$ & 8 & 0 & 100 & 8 & 0 & 100 & 10 & 0 & 100 & 26 & 0 & 100 & 0 \\
\hline $\mathrm{T} 154$ & 9 & 0 & 100 & 9 & 0 & 100 & 11 & 0 & 100 & 29 & 0 & 100 & 0 \\
\hline $\mathrm{T} 158$ & 10 & 0 & 100 & 8 & 0 & 100 & 10 & 0 & 100 & 28 & 0 & 100 & 0 \\
\hline TAM 111 & 7 & 0 & 100 & 12 & 0 & 100 & 9 & 0 & 100 & 28 & 0 & 100 & 0 \\
\hline TAM 113 & 11 & 0 & 100 & 9 & 0 & 100 & 10 & 0 & 100 & 30 & 0 & 100 & 0 \\
\hline WB4458 & 9 & 0 & 100 & 11 & 0 & 100 & 10 & 0 & 100 & 30 & 0 & 100 & 0 \\
\hline WB-Cedar & 10 & 0 & 100 & 10 & 0 & 100 & 11 & 0 & 100 & 31 & 0 & 100 & 0 \\
\hline WB-Grainfield & 10 & 0 & 100 & 9 & 0 & 100 & 12 & 0 & 100 & 31 & 0 & 100 & 0 \\
\hline Winterhawk & 11 & 0 & 100 & 11 & 0 & 100 & 9 & 0 & 100 & 31 & 0 & 100 & 0 \\
\hline LCH09-43 & 10 & 1 & 91 & 10 & 0 & 100 & 11 & 0 & 100 & 31 & 1 & 97 & 5 \\
\hline SY Gold & 8 & 0 & 100 & 9 & 1 & 90 & 11 & 0 & 100 & 28 & 1 & 97 & 8 \\
\hline SY Wolf & 6 & 1 & 86 & 9 & 0 & 100 & 11 & 1 & 92 & 26 & 2 & 93 & 7 \\
\hline TAM 304 & 11 & 0 & 100 & 7 & 2 & 78 & 10 & 0 & 100 & 28 & 2 & 93 & 13 \\
\hline TAM 401 & 8 & 2 & 80 & 11 & 0 & 100 & 10 & 1 & 91 & 29 & 3 & 91 & 10 \\
\hline Everest & 9 & 1 & 90 & 7 & 3 & 70 & 8 & 3 & 73 & 24 & 7 & 77 & 11 \\
\hline Hatcher & 6 & 3 & 67 & 2 & 8 & 20 & 3 & 8 & 27 & 11 & 19 & 37 & 25 \\
\hline GA-031257-10LE34 & 5 & 5 & 50 & 3 & 8 & 27 & 3 & 7 & 30 & 11 & 20 & 35 & 13 \\
\hline WB-Redhawk & 5 & 4 & 56 & 2 & 8 & 20 & 3 & 8 & 27 & 10 & 20 & 33 & 19 \\
\hline KSO9H19-2-3 & 4 & 6 & 40 & 1 & 4 & 20 & 3 & 8 & 27 & 8 & 18 & 30 & 10 \\
\hline Bill Brown & 0 & 9 & 0 & 3 & 7 & 30 & 0 & 11 & 0 & 3 & 27 & 10 & 17 \\
\hline Thunder CL & 0 & 8 & 0 & 2 & 8 & 20 & 1 & 10 & 9 & 3 & 26 & 10 & 10 \\
\hline Danby $(W)$ & 1 & 8 & 11 & 0 & 9 & 0 & 0 & 12 & 0 & 1 & 29 & 3 & 6 \\
\hline Brawl CL Plus & 0 & 10 & 0 & 0 & 8 & 0 & 0 & 9 & 0 & 0 & 27 & 0 & 0 \\
\hline Byrd & 0 & 8 & 0 & 0 & 8 & 0 & 0 & 12 & 0 & 0 & 28 & 0 & 0 \\
\hline Denali (Kansas) & 0 & 11 & 0 & 0 & 7 & 0 & 0 & 11 & 0 & 0 & 29 & 0 & 0 \\
\hline Endurance & 0 & 5 & 0 & 0 & 9 & 0 & 0 & 11 & 0 & 0 & 25 & 0 & 0 \\
\hline Fuller & 0 & 9 & 0 & 0 & 7 & 0 & 0 & 10 & 0 & 0 & 26 & 0 & 0 \\
\hline GA-045710-10E46 & 0 & 11 & 0 & 0 & 7 & 0 & 0 & 10 & 0 & 0 & 28 & 0 & 0 \\
\hline LCSMint & 0 & 8 & 0 & 0 & 7 & 0 & 0 & 10 & 0 & 0 & 25 & 0 & 0 \\
\hline Santa Fe & 0 & 7 & 0 & 0 & 11 & 0 & 0 & 10 & 0 & 0 & 28 & 0 & 0 \\
\hline Newton & 0 & 13 & 0 & 0 & 9 & 0 & 0 & 11 & 0 & 0 & 33 & 0 & 0 \\
\hline Karl92 & 0 & 9 & 0 & 0 & 11 & 0 & 0 & 10 & 0 & 0 & 30 & 0 & 0 \\
\hline
\end{tabular}

R, number of resistant plants; S, number of susceptible plants; T, total number of plants; \%R, percent resistant plants (mean); SD, standard deviation. Cultivars in italic were with $<80 \%$ resistant plants.

was very different on the Hessian fly resistance of different wheat cultivars. The cultivar Everest, which showed a moderate $77 \%$ resistance, remained moderately resistant at all temperatures with a slight increase in resistance at the $14-16^{\circ} \mathrm{C}$ range. Similarly, WBRedhawk, which had a low-level 33\% resistance, re- mained low, but with a slight increase at the $14-16^{\circ} \mathrm{C}$ range. Lower temperatures have no significant impact on resistance in Everest $(P=0.634)$ or WB-Redhawk $(P=0.432)$.

Conversely, lower temperatures had a dramatic impact on resistance of several cultivars. Hatcher, GA- 
Table 2. Wheat cultivars susceptible at $20-22^{\circ} \mathrm{C}$ became resistant to Hessian fly infestation at lower temperatures

\begin{tabular}{|c|c|c|c|c|c|c|c|c|}
\hline \multirow{2}{*}{ Cultivar } & \multicolumn{2}{|c|}{$14-16^{\circ} \mathrm{C}$} & \multicolumn{2}{|c|}{$16-18^{\circ} \mathrm{C}$} & \multicolumn{2}{|c|}{$18-20^{\circ} \mathrm{C}$} & \multicolumn{2}{|c|}{$20-22^{\circ} \mathrm{C}$} \\
\hline & $\mathrm{T}$ & $\% \mathrm{R} / \mathrm{SD}$ & $\mathrm{T}$ & $\% \mathrm{R} / \mathrm{SD}$ & $\mathrm{T}$ & $\% \mathrm{R} / \mathrm{SD}$ & $\mathrm{T}$ & $\% \mathrm{R} / \mathrm{SD}$ \\
\hline Everest & 33 & $88 / 7$ & 33 & $81 / 8$ & 35 & $77 / 1$ & 31 & $77 / 11$ \\
\hline Hatcher & 34 & $97 / 2$ & 33 & $76 / 4$ & 33 & $85 / 16$ & 30 & $37 / 25$ \\
\hline GA-031257-10LE34 & 33 & $91 / 9$ & 33 & $85 / 14$ & 34 & $65 / 14$ & 31 & $35 / 13$ \\
\hline Bill Brown & 34 & $97 / 5$ & 29 & $97 / 6$ & 34 & $94 / 10$ & 30 & $10 / 17$ \\
\hline Thunder CL & 34 & $94 / 5$ & 32 & $72 / 11$ & 31 & $81 / 2$ & 29 & $10 / 10$ \\
\hline Fuller & 32 & $94 / 10$ & 30 & $97 / 6$ & 34 & $71 / 2$ & 26 & $0 / 0$ \\
\hline Endurance & 35 & $94 / 10$ & 31 & $97 / 10$ & 32 & $94 / 1$ & 25 & $0 / 0$ \\
\hline Byrd & 30 & $93 / 10$ & 32 & $6 / 10$ & 31 & $3 / 6$ & 28 & $0 / 0$ \\
\hline Denali (Kansas) & 29 & $52 / 14$ & 32 & $6 / 9$ & 30 & $0 / 0$ & 29 & $0 / 0$ \\
\hline KS09H19-2-3 & 31 & $87 / 2$ & 33 & $6 / 10$ & 31 & $10 / 16$ & 26 & $30 / 10$ \\
\hline Brawl CL Plus & 34 & $18 / 5$ & 32 & $3 / 5$ & 30 & $0 / 0$ & 27 & $0 / 0$ \\
\hline GA-045710-10E46 & 30 & $40 / 9$ & 33 & $9 / 9$ & 33 & $18 / 14$ & 28 & $0 / 0$ \\
\hline Danby (W) & 35 & $43 / 9$ & 32 & $6 / 10$ & 23 & $0 / 0$ & 30 & $3 / 6$ \\
\hline LCSMint & 29 & $10 / 10$ & 31 & $17 / 10$ & 33 & $15 / 16$ & 25 & $0 / 0$ \\
\hline Santa Fe & 33 & $36 / 4$ & 32 & $31 / 11$ & 33 & $18 / 10$ & 28 & $0 / 0$ \\
\hline WB-Redhawk & 35 & $43 / 4$ & 35 & $26 / 11$ & 39 & $28 / 2$ & 30 & $33 / 19$ \\
\hline Newton & 61 & $15 / 4$ & 59 & $0 / 0$ & 56 & $0 / 0$ & 33 & $0 / 0$ \\
\hline Karl92 & 56 & $14 / 6$ & 58 & $0 / 0$ & 61 & $0 / 0$ & 30 & $0 / 0$ \\
\hline
\end{tabular}

$\mathrm{T}$, total number of plants tested; $\% \mathrm{R}$, percent resistant plants (mean); SD, standard deviation. Cultivars with $<50 \%$ resistant plants are in italic. Each treatment was repeated three times.

031257-10LE34, Bill Brown, Thunder confidence limits, Fuller, and Endurance became moderately to highly resistant (71-94\%) beginning at $18-20^{\circ} \mathrm{C}$. Byrd, Denali, and KS09H19-2-3 also became moderately to highly resistant $(52-93 \%)$ at $14-16^{\circ} \mathrm{C}$. All these differences in Hessian fly resistance were significant at the 0.05 level.

The impact of lower temperature on Brawl confidence limits Plus, 'GA-045710-10E46, Danby, LCSMint, and Santa Fe was less significant. These cultivars were susceptible at $20-22^{\circ} \mathrm{C}$, and remained susceptible or had a very low level of resistance until the temperature reached $14-16^{\circ} \mathrm{C}$, when resistance increased but did not exceed $50 \%$. The susceptible control cultivars Newton and Karl92 remained susceptible until the temperature reached $14-16^{\circ} \mathrm{C}$, when some $(<20 \%)$ plants became resistant. Lower temperature had no significant impact on resistance in LCSMint $(P=0.108)$ and Brawl confidence limits Plus $(0.004)$ to Hessian fly infestation. However, lower temperatures significantly impacted GA-045710-10E46, Danby, and Santa $\mathrm{Fe}$ at the 0.05 level, even though resistance did not exceed $50 \%$.

Some Cultivars Resistant at $\leq \mathbf{2 0}-22^{\circ} \mathrm{C}$ Become Susceptible at Higher Temperatures. Wheat cultivars with $\geq 70 \%$ resistant plants (Table 1 ) were retested at $22-24^{\circ} \mathrm{C}, 24-26^{\circ} \mathrm{C}$, and $26-28^{\circ} \mathrm{C}$ (Table 3). Everest remained moderately resistant at all temperatures. Resistance in 'TAM 401' became destabilized with only $56 \%$ resistance at $22-24^{\circ} \mathrm{C}$, and essentially lost resistance at $\geq 24^{\circ} \mathrm{C}$. Resistance in 'Clara confidence limits' became destabilized at $24-26^{\circ} \mathrm{C}$ with $<50 \%$ resistant plants, and essentially lost resistance $\geq 26^{\circ} \mathrm{C}$. 'LCH0919,' 'GA-031086-10E26,' ‘25R30,' and ‘Art', became susceptible, with resistance of $<50 \%$. 'SY Southwind,' 'SY Wolf,' 'SY Gold,' 'PostRock,' 'OK09915C,' 'Jackpot,' 'Greer,' '25R32,' and 'WB-Cedar' also became destabilized and lost resistance, with percentages of resistant plants decreasing to $50-80 \%$. Higher tempera- tures significantly impacted resistance in wheat cultivars at the 0.05 level, with the exception of SY Wolf and WB-Cedar. Other cultivars retained high levels $(>80 \%)$ of resistance at all tested temperatures.

Time Required for Phenotype Changes Under Selected Temperatures. Phenotype switching from susceptible to resistant occurred at different durations at $14-16^{\circ} \mathrm{C}$ for different cultivars (Table 4). Bill Brown and Hatcher needed only $12 \mathrm{~h}$ at $14-16^{\circ} \mathrm{C}$ to trigger resistance in $\geq 50 \%$ plants. It took $48 \mathrm{~h}$ for Thunder confidence limits, $72 \mathrm{~h}$ for Byrd and KS09H19-2-3, and $120 \mathrm{~h}$ for GA-031257-10LE34, Fuller, and Endurance to exhibit resistance in $\geq 50 \%$ plants under the same conditions.

Phenotypic Variations Among Infested Seedlings of Different Cultivars. For susceptible plants, Hessian fly feeding symptoms were rather typical (Fig. 1A and S). The elongation of the second leaf sheath was inhibited and the third leaf failed to grow out or extremely stunted if it grew out at all. Susceptible plants were all dead after larvae inside pupated (data not shown). Physical appearance varied for plants with resistance to Hessian fly infestation. Two of the most commonly observed resistant phenotypes are shown in Fig. 1. For type 1 (R1) plants, the elongation of the second leaf sheath was inhibited as observed in susceptible plants, but the third leaf grew out relatively normally $2 \mathrm{wk}$ after Hessian fly infestation (Fig. 1A and R1 in the middle). Elongation of the third leaf sheath and growth of fourth leaf were normal in type 1 plants when the plants were continuously cultured in growth chambers (Fig. 1, R1 on the right). For type 2 plants (R2), elongation of the second leaf sheath and growth of third leaf were similar to uninfested control plants (Fig. 1, R2). Fly larvae in resistant plants were dead in resistant plants without apparent growth or development, whereas larvae in susceptible plants developed into second instars $2 \mathrm{wk}$ after infestation (Fig. 1B). 
Table 3. Wheat cultivars with Hessian fly resistance at $20-22^{\circ} \mathrm{C}$ lose resistance at higher temperatures

\begin{tabular}{|c|c|c|c|c|c|c|c|c|}
\hline \multirow{2}{*}{ Cultivar } & \multicolumn{2}{|c|}{$20-22^{\circ} \mathrm{C}$} & \multicolumn{2}{|c|}{$22-24^{\circ} \mathrm{C}$} & \multicolumn{2}{|c|}{$24-26^{\circ} \mathrm{C}$} & \multicolumn{2}{|c|}{$26-28^{\circ} \mathrm{C}$} \\
\hline & $\mathrm{T}$ & $\% \mathrm{R} / \mathrm{SD}$ & $\mathrm{T}$ & $\% \mathrm{R} / \mathrm{SD}$ & $\mathrm{T}$ & $\% \mathrm{R} / \mathrm{SD}$ & $\mathrm{T}$ & $\% \mathrm{R} / \mathrm{SD}$ \\
\hline TAM 401 & 32 & $91 / 10$ & 34 & $56 / 16$ & 34 & $23 / 3$ & 36 & $16 / 8$ \\
\hline Clara CL (W) & 29 & $100 / 0$ & 33 & $82 / 9$ & 33 & $43 / 7$ & 32 & $18 / 8$ \\
\hline LCH09-19 & 30 & $100 / 0$ & 32 & $100 / 0$ & 28 & $97 / 5$ & 31 & $10 / 8$ \\
\hline GA-031086-10E26 & 33 & $100 / 0$ & 29 & $100 / 0$ & 27 & $93 / 6$ & 31 & $15 / 15$ \\
\hline $25 \mathrm{R} 30$ & 27 & $100 / 0$ & 31 & $100 / 0$ & 30 & $90 / 9$ & 31 & $27 / 15$ \\
\hline Art & 34 & $100 / 0$ & 31 & $97 / 6$ & 29 & $83 / 4$ & 28 & $48 / 21$ \\
\hline SY Southwind & 28 & $100 / 0$ & 33 & $92 / 8$ & 28 & $61 / 6$ & 31 & $50 / 13$ \\
\hline SY Wolf & 28 & $93 / 7$ & 33 & $88 / 3$ & 34 & $77 / 25$ & 30 & $56 / 13$ \\
\hline SY Gold & 29 & $97 / 6$ & 33 & $85 / 4$ & 33 & $82 / 2$ & 32 & $57 / 16$ \\
\hline PostRock & 29 & $100 / 0$ & 30 & $100 / 0$ & 34 & $92 / 8$ & 27 & $59 / 8$ \\
\hline OK09915C & 30 & $100 / 0$ & 29 & $100 / 0$ & 32 & $88 / 4$ & 30 & $60 / 6$ \\
\hline Jackpot & 30 & $100 / 0$ & 27 & $100 / 0$ & 28 & $94 / 6$ & 28 & $62 / 11$ \\
\hline Greer & 30 & $100 / 0$ & 32 & $100 / 0$ & 32 & $94 / 5$ & 35 & $63 / 3$ \\
\hline Everest & 31 & $77 / 11$ & 27 & $66 / 4$ & 31 & $77 / 6$ & 32 & $72 / 10$ \\
\hline $25 \mathrm{R} 32$ & 29 & $100 / 0$ & 30 & $100 / 0$ & 31 & $100 / 0$ & 31 & $74 / 10$ \\
\hline WB-Cedar & 31 & $100 / 0$ & 26 & $100 / 0$ & 33 & $92 / 8$ & 33 & $79 / 3$ \\
\hline $25 \mathrm{R} 39$ & 29 & $100 / 0$ & 26 & $100 / 0$ & 30 & $97 / 6$ & 28 & $81 / 17$ \\
\hline TAM 111 & 28 & $100 / 0$ & 32 & $100 / 0$ & 33 & $86 / 8$ & 34 & $82 / 9$ \\
\hline WB-Grainfield & 31 & $100 / 0$ & 27 & $100 / 0$ & 30 & $97 / 4$ & 30 & $94 / 5$ \\
\hline Winterhawk & 31 & $100 / 0$ & 26 & $100 / 0$ & 30 & $100 / 0$ & 31 & $94 / 5$ \\
\hline Armour & 26 & $100 / 0$ & 28 & $100 / 0$ & 28 & $97 / 4$ & 27 & $96 / 6$ \\
\hline $\mathrm{T} 158$ & 28 & $100 / 0$ & 32 & $100 / 0$ & 27 & $100 / 0$ & 31 & $97 / 6$ \\
\hline TAM 304 & 30 & $93 / 13$ & 27 & $100 / 0$ & 26 & $97 / 5$ & 29 & $97 / 6$ \\
\hline WB4458 & 30 & $100 / 0$ & 33 & $97 / 4$ & 30 & $97 / 5$ & 29 & $97 / 5$ \\
\hline 1863 & 29 & $100 / 0$ & 27 & $100 / 0$ & 25 & $100 / 0$ & 30 & $97 / 5$ \\
\hline CJ & 32 & $100 / 0$ & 24 & $100 / 0$ & 26 & $100 / 0$ & 28 & $97 / 5$ \\
\hline Gallagher & 32 & $100 / 0$ & 26 & $100 / 0$ & 29 & $100 / 0$ & 29 & $97 / 5$ \\
\hline LCH08-80 & 32 & $100 / 0$ & 25 & $100 / 0$ & 29 & $100 / 0$ & 26 & $97 / 5$ \\
\hline Billings & 34 & $100 / 0$ & 26 & $100 / 0$ & 29 & $100 / 0$ & 33 & $97 / 4$ \\
\hline LCH08-109 & 29 & $100 / 0$ & 31 & $100 / 0$ & 28 & $100 / 0$ & 26 & $100 / 0$ \\
\hline LCH09-43 & 32 & $97 / 5$ & 26 & $100 / 0$ & 26 & $100 / 0$ & 25 & $100 / 0$ \\
\hline LCS08-12 & 30 & $100 / 0$ & 32 & $100 / 0$ & 32 & $100 / 0$ & 32 & $100 / 0$ \\
\hline $\mathrm{T} 153$ & 26 & $100 / 0$ & 27 & $100 / 0$ & 26 & $100 / 0$ & 27 & $100 / 0$ \\
\hline $\mathrm{T} 154$ & 29 & $100 / 0$ & 30 & $100 / 0$ & 29 & $100 / 0$ & 30 & $100 / 0$ \\
\hline 2135 & 34 & $100 / 0$ & 28 & $100 / 0$ & 29 & $100 / 0$ & 31 & $100 / 0$ \\
\hline 2166 & 29 & $100 / 0$ & 29 & $100 / 0$ & 31 & $100 / 0$ & 28 & $100 / 0$ \\
\hline 2525 & 29 & $100 / 0$ & 24 & $100 / 0$ & 23 & $100 / 0$ & 29 & $100 / 0$ \\
\hline Centerfield & 27 & $100 / 0$ & 29 & $100 / 0$ & 30 & $97 / 6$ & 25 & $100 / 0$ \\
\hline Deliver & 27 & $100 / 0$ & 27 & $100 / 0$ & 34 & $97 / 5$ & 31 & $100 / 0$ \\
\hline Duster & 29 & $100 / 0$ & 29 & $100 / 0$ & 29 & $100 / 0$ & 28 & $100 / 0$ \\
\hline Garrison & 26 & $100 / 0$ & 32 & $100 / 0$ & 29 & $100 / 0$ & 26 & $100 / 0$ \\
\hline TAM 113 & 30 & $100 / 0$ & 30 & $100 / 0$ & 33 & $97 / 4$ & 28 & $100 / 0$ \\
\hline Newton & 33 & $0 / 0$ & 34 & $0 / 0$ & 33 & $0 / 0$ & 34 & $0 / 0$ \\
\hline Karl92 & 30 & $0 / 0$ & 34 & $0 / 0$ & 37 & $0 / 0$ & 37 & $0 / 0$ \\
\hline
\end{tabular}

$\mathrm{T}$, total number of plants tested; \%R, percent resistant plants (mean); SD, standard deviation.

Cultivars with $<50 \%$ resistant plants are in italic, or $80 \%$ resistant plants are in bold. Each treatment was repeated three times.

Table 4. Times (h) exposed to $14-16^{\circ} \mathrm{C}$ for wheat cultivars to exhibit Hessian fly resistance at $20^{\circ} \mathrm{C}$

\begin{tabular}{|c|c|c|c|c|c|c|c|c|c|c|}
\hline \multirow{2}{*}{ Cultivar } & \multicolumn{2}{|c|}{$12 \mathrm{~h}$} & \multicolumn{2}{|c|}{$24 \mathrm{~h}$} & \multicolumn{2}{|c|}{$48 \mathrm{~h}$} & \multicolumn{2}{|c|}{$72 \mathrm{~h}$} & \multicolumn{2}{|c|}{$120 \mathrm{~h}$} \\
\hline & $\mathrm{T}$ & $\% \mathrm{R} / \mathrm{SD}$ & $\mathrm{T}$ & $\% \mathrm{R} / \mathrm{SD}$ & $\mathrm{T}$ & $\% \mathrm{R} / \mathrm{SD}$ & $\mathrm{T}$ & $\% \mathrm{R} / \mathrm{SD}$ & $\mathrm{T}$ & $\% \mathrm{R} / \mathrm{SD}$ \\
\hline Bill Brown & 32 & $50 / 9$ & 32 & $97 / 5$ & 32 & $97 / 5$ & 29 & $93 / 6$ & 34 & $97 / 5$ \\
\hline Hatcher & 34 & $71 / 6$ & 34 & $76 / 5$ & 31 & $68 / 2$ & 32 & $94 / 10$ & 34 & $97 / 2$ \\
\hline Thunder CL & 26 & $27 / 16$ & 30 & $27 / 2$ & 31 & $52 / 8$ & 31 & $97 / 6$ & 34 & $94 / 5$ \\
\hline Byrd & 30 & $0 / 0$ & 30 & $0 / 0$ & 32 & $6 / 10$ & 32 & $75 / 11$ & 30 & $93 / 10$ \\
\hline KS09H19-2-3 & 29 & $14 / 12$ & 29 & $17 / 14$ & 34 & $12 / 10$ & 35 & $86 / 6$ & 31 & $87 / 2$ \\
\hline GA-031257-10LE34 & 33 & $30 / 5$ & 31 & $39 / 7$ & 32 & $34 / 7$ & 36 & $36 / 5$ & 33 & $91 / 9$ \\
\hline Fuller & 32 & $3 / 5$ & 30 & $3 / 5$ & 29 & $3 / 6$ & 33 & $30 / 3$ & 32 & $94 / 10$ \\
\hline Endurance & 30 & $3 / 6$ & 32 & $3 / 5$ & 32 & $6 / 10$ & 29 & $31 / 6$ & 35 & $94 / 10$ \\
\hline Newton & 56 & $0 / 0$ & 64 & $5 / 5$ & 58 & $10 / 9$ & 61 & $10 / 4$ & 61 & $15 / 4$ \\
\hline Karl92 & 59 & $2 / 3$ & 60 & $5 / 8$ & 60 & $10 / 6$ & 55 & $15 / 4$ & 56 & $14 / 6$ \\
\hline
\end{tabular}

Seedlings infested with Hessian fly larvae were continuously cultivated at $14-16^{\circ} \mathrm{C}$ for $12,24,48,72$, and $120 \mathrm{~h}$, and were then transferred to $20^{\circ} \mathrm{C}$ for phenotyping.

Italics indicate cultivars with $>50 \%$ resistant plants under that duration exposed to $14-16^{\circ} \mathrm{C}$. Each treatment was repeated three times.

$\mathrm{T}$, total number of plants tested; \%R, percent resistant plants (mean); SD, standard deviation. 
A

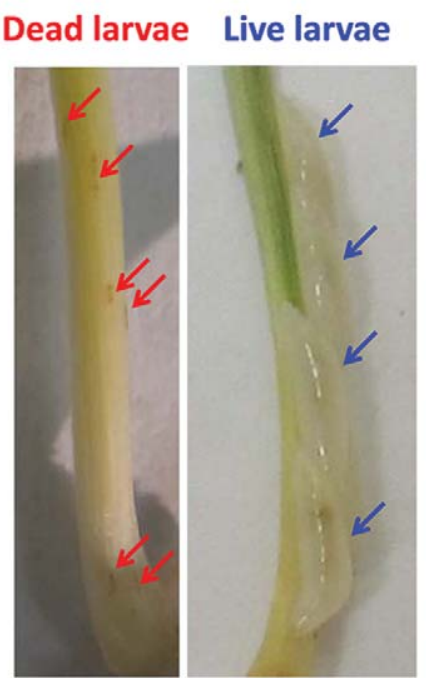

B

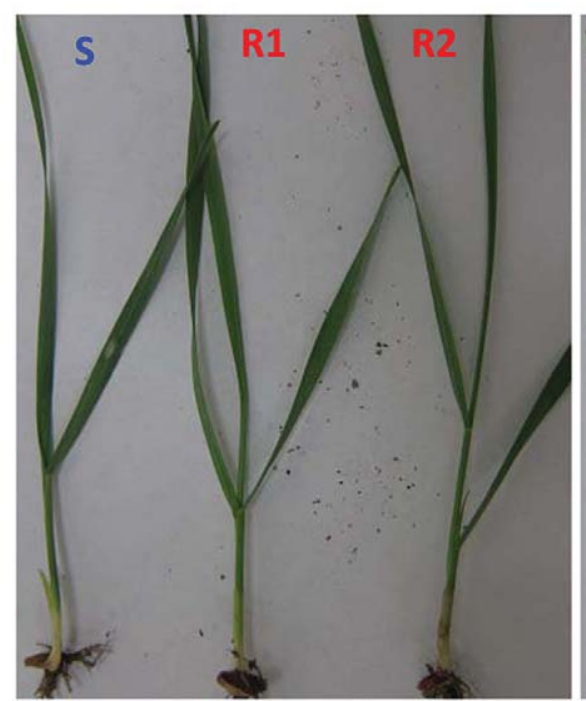

Fig. 1. Variations in phenotypes of susceptible and resistant wheat seedlings. (A) Skinny dead larvae (indicated by red arrows on the left side) in resistant plants, and fat live larvae (indicated by blue arrows on the right) in susceptible plants. (B) Plant appearance of susceptible and resistant plants after Hessian fly attack. The plant indicated by the letter S represents the appearance of a typical susceptible plant $2 \mathrm{wk}$ after the initial Hessian fly attack. The elongation of the second leaf sheath is inhibited, and often the third leaf fails to grow out. The plant indicated by $\mathrm{R} 1$ in the middle represents the appearance of wheat seedlings of some resistant cultivars $2 \mathrm{wk}$ after Hessian fly attack. The elongation of the second leaf sheath was inhibited, but the third leaf grows out. After another week, the plant indicated by R1 in the middle develops into a plant indicated by $\mathrm{R} 1$ on the right, with elongation of the third leaf sheath and growth of the fourth leaf. The plant indicated by R2 is a different type of appearance of resistant seedlings. The plant grows relatively normally with elongation of the second leaf sheath and growth of the third leaf.

Most cultivars tested with resistance to Hessian fly at low temperatures $\left(<20^{\circ} \mathrm{C}\right)$ had the type 1 phenotype (Bill Brown, Denali, Fuller, GA-031257-10LE34, Hatcher, and Thunder confidence limits; Fig. 2). Exceptions were Byrd and Endurance, which exhibited the type 2 phenotype. In contrast, most wheat cultivars with Hessian fly resistance at $\geq 20^{\circ} \mathrm{C}$ exhibited the type 2 phenotype, but there were also exceptions (Fig. 2).

\section{Discussion}

The impact of temperature on various plant traits attracts a great deal of attention in both the public and the scientific community because of climate change. The impact of temperature on the effectiveness of wheat resistance to Hessian fly is particularly important for the management of this insect pest for several reasons. First, the loss of wheat resistance to Hessian

\begin{tabular}{l|lll}
$\begin{array}{l}\text { Type 1 Resistance } \\
\text { Phenotype (R1) }\end{array}$ & \multicolumn{3}{|l}{$\begin{array}{l}\text { Type 2 Resistance } \\
\text { Phenotype (R2) }\end{array}$} \\
\hline Art & 1863 & Duster & SY Southwind \\
Bill Brown & 2135 & Endurance & SY Wolf \\
Clara CL & 2166 & GA-031086- & T153 \\
Denali & 2525 & $10 E 26$ & T154 \\
Everest & $25 R 30$ & Garrison & T158 \\
Fuller & $25 R 32$ & Greer & TAM 111 \\
GA-031257-10LE34 & $25 R 39$ & Jackpot & TAM 113 \\
Gallagher & Armour & LCH08-80 & TAM 304 \\
Hatcher & Billings & LCH09-19 & WB4458 \\
LCH08-109 & Byrd & LCH09-43 & WB-Cedar \\
OK09915C & Centerfield & LCS08-12 & WB-Grainfield \\
TAM 401 & CJ & PostRock & Winterhawk \\
Thunder CL & Deliver & SY Gold &
\end{tabular}

Fig. 2. Wheat cultivars with the two types of phenotypic appearance of resistant plants after Hessian fly attack. The bold and shaded cultivars were resistant to Hessian fly infestation only under low $\left(<20^{\circ} \mathrm{C}\right)$ temperatures. 

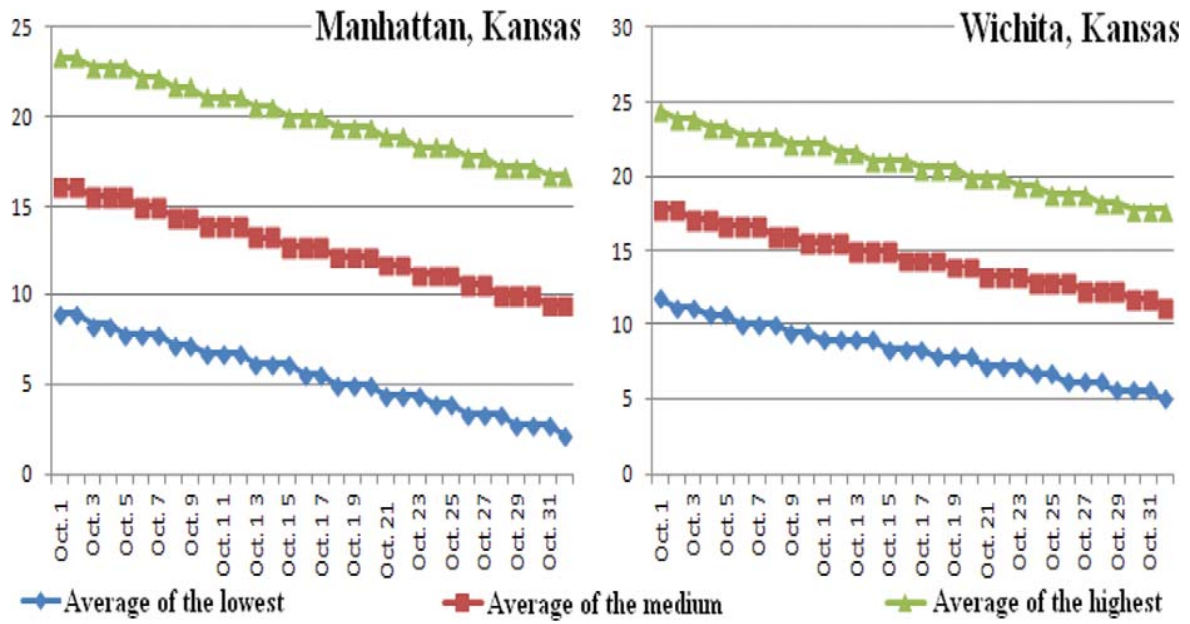

Fig. 3. The 2002-2012 temperatures in October in Manhattan and Wichita, KS, from the National Weather Service center of the National Oceanic and Atmospheric Administration (http://www.weatherpages.com/wxhistory.html).

fly at higher temperatures poses a threat to the overall strategy of using plant resistance for controlling this pest in a global warming scenario. Historically, plant resistance has played a crucial role in reducing wheat yield loss due to Hessian fly infestation (Buntin et al. 1992). Second, even a slight temperature change that results in a resistant plant becoming susceptible or vice versa presented a major challenge in breeding Hessian fly-resistant wheat and in basic research to understand the Hessian fly resistance mechanism. Greenhouse evaluation of breeding lines for fly resistance often yields inconsistent results from year to year, and these results also complicate efforts to map and clone Hessian fly resistance genes in wheat. Third, the existence of a wide range of wheat cultivars with resistance at lower temperatures was unknown, and the impact of this type of resistance on Hessian fly population dynamics remains to be evaluated.

Historically, the resistance strategy for controlling Hessian fly has overall been less successful in the southern United States than in the northern United States. One hypothesis for this phenomenon is that Hessian fly has more generations in the South, and therefore may overcome plant resistance more quickly. According to this study and previous reports (Buntin et al. 1990), the differences in temperatures during the wheat growing seasons between the South and the North could be another major factor. For example, the historical average temperatures in October in the Kansas City (MO) area is $9-19^{\circ} \mathrm{C}$ (medium average at the end of October-medium average at the beginning of October), whereas that in the Dallas (TX) area is $14-25^{\circ} \mathrm{C}$, according to the National Weather Service center of the National Oceanic and Atmospheric Administration (http://www.weatherpages. com/wxhistory.html). These $5-6^{\circ} \mathrm{C}$ differences mean that many wheat cultivars that are resistant to Hessian fly infestation in Kansas may become susceptible or less resistant in Texas. The impact of temperature on the effectiveness of controlling Hessian fly damage using resistant wheat cultivars needs to be fully evaluated from region to region.

Many wheat cultivars popular in the Great Plains area, including Bill Brown, Byrd, Fuller, and Endurance, were classified as susceptible to the Hessian fly by traditional standards. Yet these cultivars become fully resistant to Hessian fly infestation at temperatures below $14-20^{\circ} \mathrm{C}$, depending on the specific cultivar (Table 2). Historically, average medium temperatures are $10-15^{\circ} \mathrm{C}$ in Kansas (Fig. 3), which is in the right range for these wheat cultivars to exhibit Hessian fly resistance. The existence of cultivars resistant to Hessian $\mathrm{fly}<20^{\circ} \mathrm{C}$ in the Great Plains may have played an unrecognized role in controlling this pest. The potential benefit of wheat cultivars with resistance at lower temperatures needs to be investigated.

Wheat resistance to Hessian fly is currently thought to be conferred by major dominant resistance genes with antibiosis (Stuart et al. 2012). These resistance genes interact with Hessian fly avirulence genes in a typical gene-for-gene fashion. The genetic mechanism of wheat resistance to Hessian fly infestation at low temperatures is not yet known. Phenotypically, most cultivars with resistance at the lower temperature ranges appear to have a greater defense cost in terms of wheat growth inhibition (Figs. 1 and 2), which could indicate a different resistance mechanism. It remains to be determined if single major genes or multiple minor genes are responsible for Hessian fly resistance at lower temperatures. Further investigation into the genetic and molecular mechanisms involved in low temperature-associated resistance is needed, and could lead to new ways to generate more durable resistance of wheat cultivars for Hessian fly management.

\section{Acknowledgment}

We thank C. Michael Smith for reviewing an earlier version of the manuscript, Drs. Jerry Johnson, Marla Hall, Sid 
Perry, Brett Carver, and Guorong Zhang for allowing us to use their new wheat cultivars for this study before their official release, and Shauna Dendy for technical assistance. This research was partially supported by the grant U.S. Department of Agriculture-National Institute of Food and Agriculture-Risk Avoidance and Mitigation program (USDANIFA-RAMP) \#2010-51101-21642. Mention of trade names or commercial products in this publication is solely for the purpose of providing specific information and does not imply recommendation or endorsement by the USDA. USDA is an equal opportunity provider and employer.

\section{References Cited}

Amri, A., T. S. Cox, J. H. Hatchett, and B. S. Gill. 1990. Complementary action of genes for Hessian fly resistance in the wheat cultivar 'Seneca'. J. Hered. 81: 224-227.

Bonferroni, C. E. 1935. Il calcolo delle assicurazioni su gruppi di teste, pp. 13-60. In Studi in Onore del Professore Salvatore Ortu Carboni. Rome: Italy.

Buntin, G. D. 1992. Assessment of a microtube injection system for applying systemic insecticides at planting for Hessian fly control in winter wheat. Crop Prot. 11: 366 370.

Buntin, G. D. 1999. Hessian fly (Diptera: Cecidomyiidae) injury and loss of winter wheat grain yield and quality. J. Econ. Entomol. 92: 1190-1197.

Buntin, G. D., and P. L. Bruckner. 1990. Effect of planting date on Hessian fly infestation and production of triticale. Appl. Agric. Res. 5: 82-88.

Buntin, G. D., P. L. Bruckner, J. W. Johnson, J. E. Foster. 1990. Effectiveness of selected genes for Hessian fly resistance in wheat. J. Agric. Entomol. 7: 283-291.

Buntin, G. D., S. L. Ott, and J. W. Johnson. 1992. Integration of plant resistance, insecticides, and planting date for management of the Hessian fly (Diptera: Cecidomyiidae) in winter wheat. J. Econ. Entomol. 85: 530-538.

Byers, R. A., and R. L. Gallun. 1971. Ability of the Hessian fly to stunt winter wheat. I. Effect of larval feeding on elongation of leaves. J. Econ. Entomol. 65: 955-958.

Cambron, S. E., H. W. Ohm, R. H. Ratcliffe, and F. L. Patterson. 1996. A second gene for resistance to Hessian fly in Iumillo durum wheat. Crop Sci. 36: 1099-1101.

Comis, D. 2007. Virulent Hessian flies renew attack on U.S. wheat. United States Department of Agriculture Agricultural Research - News and Events (January 24). (http:// www.ars.usda.gov/is/pr/2007/070124.htm).

Colyer, P. D., P. R. Vernon, and T. Riley. 1989. First report of Hessian fly (Diptera: Cecidomyiidae) on winter wheat in Louisiana. J. Agric. Entomol. 7: 147-148.

Gagne, R. J., and J. H. Hatchett. 1989. Instars of the Hessian fly (Diptera: Cecidomyiidae). Ann. Entomol. Soc. Am. 82: 73-79.

Gould, F. 1998. Sustainability of transgenic insecticidal cultivars: integrating pest genetics and ecology. Annu. Rev. Entomol. 43: 701-726.
Hatchett, J. H., K. J. Starks, and J. A. Webster. 1987. Insect and mite pests of wheat, pp. 625-675. Wheat and Wheat Improvement. Agronomy Monograph No. 13. American Society of Agronomy, Madison, WI.

Huang, F., S. Harrison, B. R. Leonard, M. Ghimire, and P. P. Price. 2011. Hessian fly, an emerging wheat pest in Louisiana. Louisiana Agriculture Mag. (http://text. lsuagcenter.com/en/communications/publications / agmag/Archive/2011/Winter/Hessian-Fly.htm).

Knutson, A., and J. Swart. 2007. Hessian fly outbreaks showing up in many north Texas wheat fields. Southwest Farm Report. (http://southwestfarmpress.com/grains/041807hessian-fly/).

Li, C., M. S. Chen, S. Chao, J. Yu, and G. Bai. 2013. Identification of a novel gene, H34, in wheat using recombinant inbred lines and single nucleotide polymorphism markers. Theor. Appl. Genet. (in press).

Pauly, P. J. 2002. Fighting the Hessian fly. Environ Hist. 7: 385-507.

Ratanatham, S., and R. L.Gallun. 1986. Resistance to Hessian fly (Diptera: Cecidomyiidae) in wheat as affected by temperature and larval density. Environ. Entomol. 15: 305-310.

Ratcliffe, R. H., G. G. Safranski, F. L. Patterson, H.W. Ohm, and P. L. Taylor. 1994. Biotype status of Hessian fly (Diptera: Cecidomyiidae) populations from the eastern United States and their response to 14 Hessian fly resistance genes. J. Econ. Entomol. 87: 1113-1121.

Royer, T. A. 2005. Hessian fly reported in Oklahoma wheat. (http://www.ento.okstate.edu/Pddl/2005/PDIA41.pdf).

Smith, R. 2007. Widespread outbreaks of Hessian fly reported in wheat. Southwest Farm Report. (http:// southwestfarmpress.com/grains/051807-hessian-fly/).

Sosa, O., Jr. 1979. Hessian fly resistance of wheat as affected by temperature and duration of exposure. Environ. Entomol. 8: 280-281.

Sosa, O., Jr., and J. E. Foster. 1976. Temperature and the expression of resistance in wheat to the Hessian fly. Environ. Entomol. 5: 333-336.

Stuart, J. J., M. S. Chen, M. O. Harris, and R. H. Shukle. 2012. Gall midges (Hessian Flies) as plant pathogens. Annu. Rev. Phytopathol 50: 339-357.

Tyler, J. M., and J. H. Hatchett. 1983. Temperature influence on expression of resistance to Hessian fly (Diptera: Cecidomyiidae) in wheat derived from Triticum taushii. J. Econ. Entomol. 76: 323-326.

Watson, S. 2005. Hessian fly problems have been increasing in recent years in the Central Plains. Wheat Farmer/Row Crop Farmer 9: 4-5.

Zelarayan, E. L., G. D. Buntin, J. W. Johnson, P. L. Bruckner, and P. L. Raymer. 1991. Integrated management for Hessian fly in Triticale. J. Prod. Agric. 4: 629-633.

Received 10 August 2013; accepted 21 March 2014. 